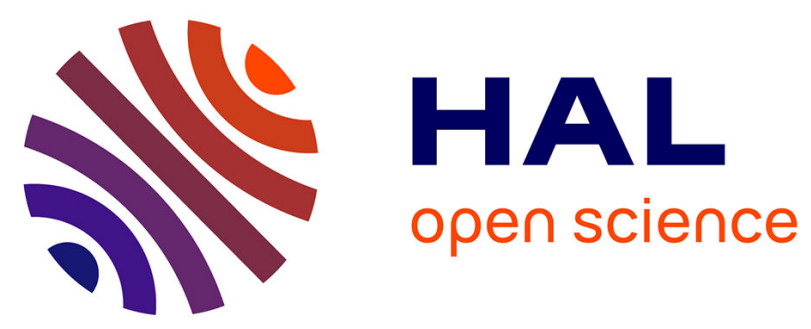

\title{
NON-PREMIXED TURBULENT COMBUSTION MODELLING OF A BLUFF-BODY FLAME USING A FLAMELET PROGRESS VARIABLE APPROACH
}

Javier Manrique, Cesar Celis, Luis Fernando Figueira da Silva

\section{- To cite this version:}

Javier Manrique, Cesar Celis, Luis Fernando Figueira da Silva. NON-PREMIXED TURBULENT COMBUSTION MODELLING OF A BLUFF-BODY FLAME USING A FLAMELET PROGRESS VARIABLE APPROACH. 26th International Congress of Mechanical Engineering, Nov 2021, Florianopolis, Brazil. 10.26678/ABCM.COBEM2021.COB2021-0080 . hal-03322357

\section{HAL Id: hal-03322357 https://hal.science/hal-03322357}

Submitted on 19 Aug 2021

HAL is a multi-disciplinary open access archive for the deposit and dissemination of scientific research documents, whether they are published or not. The documents may come from teaching and research institutions in France or abroad, or from public or private research centers.
L'archive ouverte pluridisciplinaire HAL, est destinée au dépôt et à la diffusion de documents scientifiques de niveau recherche, publiés ou non, émanant des établissements d'enseignement et de recherche français ou étrangers, des laboratoires publics ou privés. 


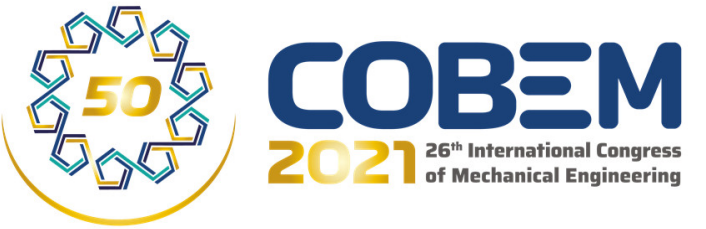

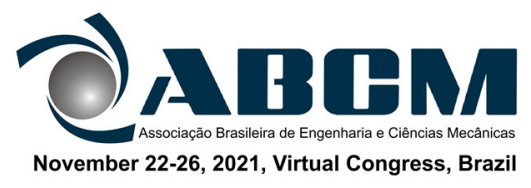

\section{COB-2021-0080 \\ NON-PREMIXED TURBULENT COMBUSTION MODELLING OF A BLUFF-BODY FLAME USING A FLAMELET PROGRESS VARIABLE APPROACH}

\section{Javier Manrique, Cesar Celis}

Mechanical Engineering Section, Pontificia Universidad Católica del Perú

Av. Universitaria 1801, San Miguel, Lima 32, Lima, Peru

edward.manrique@pucp.edu.pe, ccelis@pucp.edu.pe

\section{Luís Fernando Figueira da Silva}

Deparment of Mechanical Engineering, Pontifícia Universidade Católica do Rio de Janeiro

Rua Marquês de São Vicente 225, Rio de Janeiro, RJ 22451-900, Brazil

luisfer@puc-rio.br

Abstract. As part of the development of a numerical framework to study soot formation in turbulent combustion processes, a flamelet progress variable (FPV) combustion model is utilized here to perform numerical simulations of turbulent reacting flows. More specifically, a non-premixed turbulent flame of methane-air stabilized in a semi-infinite bluff-body burner is numerically studied using OpenFOAM. The selected axisymmetric configuration consists of a central jet of $\mathrm{CH}_{4}$ in a cylindrical bluff-body and an outer coflowing-air stream. The interaction between chemical reactions and turbulence is described with the FPV combustion model and finite-rate chemistry effects are accounted for through the detailed chemical kinetic mechanism GRI-MECH 3.0. The flamelet library is built up in the preprocessing step using a laminar counterflow diffusion flame configuration, following specific criteria carefully chosen so as to properly describe several flame conditions ranging from long enough flow residence times to flame extinction limits. All numerical simulations are performed in a large eddy simulation (LES) based context. For the LES studies, the wall-adapting local eddy-viscosity (WALE) model is used as subgrid-scale one. Numerical results are compared with an experimental data set including velocity field and $\mathrm{OH}$ radical fluorescence obtained by joint particle image velocimetry (PIV) and planar laser induced fluorescence (PLIF). The comparisons between the obtained numerical results and the corresponding experimental data include mean fields of velocity, Reynolds stress tensors, turbulent kinetic energy and normalized $\mathrm{OH}$. Overall, a relatively good agreement between numerical predictions and experimental results was observed. Both the double vortex structure and the recirculation zone length were in particular well predicted. In addition, Reynolds stresses and turbulent kinetic energy predictions agree relatively well with the experiment. However, predicted normalized $\mathrm{OH}$ radical profiles presented significantly discrepancies when compared to the experiments due to the lifted nature of the measured flame was not well predicted.

Keywords: Turbulent non-premixed flames, Bluff-body, FPV model, LES, OpenFOAM

\section{INTRODUCTION}

Nowadays approximately $80 \%$ of consumed global energy comes from combustion processes. In addition, compared to the amount corresponding to 2011, global energy demand is expected to increase near one-third by 2040. However, in spite of the rapid growth in the use of renewable energies, fossil fuels - oil, gas and coal - will remain the main sources for global energy supply in the next few years (Randers, 2012). However, due to the associated emission of greenhouse gases, particulate matter and soot, the wide use of combustion systems has a significant impact on both health and environment (Bourdrel, 2017). Therefore, in order to increase fuel efficiency and to reduce pollutant emissions, continuous design improvements in combustion systems need to be introduced.

Bluff-body burner like configurations featuring strong turbulence-chemistry interactions are typically used as a means of flame stabilization in both gas turbine combustors and industrial burners. The characteristic recirculating flow regions formed in such configurations have a significant influence on both flame stabilization mechanisms and residence time distributions, which subsequently affect soot and NOx formation in flames (Rowhani et al., 2020). Previous studies have shown that the peak of soot volume fraction is associated with the low strain rates, high temperature regions of the flame, whereas advection processes can explain the presence of soot in high local strain rates shear layer regions unfavorable for soot to form (Mueller et al., 2013). Both time and length scales characterizing turbulent reacting flows control indeed the pollutant formation in flames. An adequate treatment of the turbulence-chemistry interactions usually present in bluffbody burner configurations is therefore needed in order to improve existing combustion systems. 
Combustion processes and flame behavior in particular are highly sensitive to inflow conditions (Peters, 2000). This means that accurate modelling of turbulent transport of momentum, energy and species mass is paramount to obtaining reliable simulation results. Non-reactive and reactive flow studies show that, compared to Reynolds-averaged NavierStokes (RANS) approaches, large-eddy simulations (LES) based ones predict scalar mixing processes and dissipation rates with considerably improved accuracy, especially in complex flows (Pitsch, 2006). In this work in particular, the wall-adapting local eddy viscosity (WALE) model is used as sub-grid scale (SGS) one to close the LES equations. For turbulent bluff-body related flows, WALE model has been shown to capture general flow features including the shear layer, sudden expansion and recirculation zone (Lee and Cant, 2016).

Regarding combustion modeling, a finite-rate chemistry combustion model, i.e., the flamelet/progress variable (FPV) (Pierce and Moin, 2004) one, is employed here. The non-premixed flame structure is a priori computed in mixture fraction space by solving the steady flamelet equations (Peters, 2000). In order to consider all steady flamelet solutions, a reaction progress parameter, denoted by $C$, has been introduced in the FPV model. This parameter identifies each single flame state including both stable and unstable solutions and it is represented here by a linear combination of major reaction product mass fractions. In this work therefore, all thermochemical quantities are parameterized with a reduced set of variables (mixture fraction $Z$, progress variable $C$ ) and stored in a database that is accessed during LES simulations.

The main objective of this work is to assess the FPV model capabilities to predict turbulent non-premixed flames in bluff-body burner configurations featuring strong turbulence-chemistry interactions. In addition, it is of particular interest to determine whether the modeling approaches employed are able to capture the fundamental reactive flow features characterizing the studied burners. The numerical results obtained here are compared with those measured in a flame featuring a wake-dominated flow field with intense turbulence where intermittent flame lift-off and partial extinctions may occur (Caetano and Figueira da Silva, 2015). All numerical simulations are carried out using the open source computational tool OpenFOAM, version v2006 (OpenCFD, 2020). Due to the strong coupling between chemistry, turbulence and soot formation, the combustion model has a significant impact on soot predictions (Valencia et al., 2021). Accordingly, this work represents an additional effort towards the long-term goal involving the development of a numerical framework to properly describe soot formation in turbulent combustion processes. This work is organized as follows. Sections 2 and 3 describe, respectively, the mathematical and numerical modeling approaches employed here. In Section 4 the main results are presented and discussed, in both qualitative and quantitative terms. Finally, Section 5 summarizes the main conclusions drawn from the results obtained here.

\section{MATHEMATICAL MODEL}

The main features of the mathematical model utilized in this work are briefly described in this section. A particular emphasis is put on the flow governing equations and the combustion modeling approach employed here.

\subsection{LES governing equations}

In LES approaches, coherent large-scale structures of the turbulent flow are computationally resolved, and the effects of smaller and numerically unresolved scales on the large ones are modeled. The decomposition of scales is carried out by applying a low-pass filter to the flow field quantities. The Favre-filtered equations describing the conservation of mass and momentum are written thus as (Poinsot and Veynante, 2005),

$$
\begin{aligned}
& \frac{\partial \bar{\rho}}{\partial t}+\frac{\partial \bar{\rho} \tilde{u}_{j}}{\partial x_{j}}=0 \\
& \frac{\partial \bar{\rho} \tilde{u}_{i}}{\partial t}+\frac{\partial\left(\bar{\rho} \tilde{u}_{i} \tilde{u}_{j}\right)}{\partial x_{j}}=-\frac{\partial \bar{p}}{\partial x_{i}}+\frac{\partial \tilde{\sigma}_{i j}}{\partial x_{i}}+\frac{\partial \sigma_{i j}^{s g s}}{\partial x_{i}}, \text { for } i \text { and } j=1,2,3
\end{aligned}
$$

where $\bar{\rho}$ stands for filtered density, $\bar{p}$ is the filtered pressure, $\tilde{u}$ is the filtered velocity, and $\tilde{\sigma}_{i j}$ is the filtered viscous stress tensor. In this work, this last parameter is computed from,

$$
\tilde{\sigma}_{i j}=2 \mu\left(\tilde{S}_{i j}-\frac{1}{3} \delta_{i j} \tilde{S}_{k k}\right) \text { with } \tilde{S}_{i j}=\frac{1}{2}\left(\frac{\partial \widetilde{u}_{i}}{\partial x_{j}}+\frac{\partial \widetilde{u}_{j}}{\partial x_{i}}\right),
$$

where $\mu$ is the dynamic viscosity. The subgrid-scale (SGS) stress tensor, $\sigma_{i j}^{s g s}=\bar{\rho}\left(\tilde{u}_{i} \tilde{u}_{j}-\widetilde{u_{l} u_{j}}\right.$ ), appears in unclosed form, and it is modeled here using the WALE model (Nicoud and Ducros, 1999).

\subsection{Combustion modeling}

The flamelet/progress variable (FPV) model (Pierce and Moin, 2004; Ihme and Pitsch, 2005) employed in this work is based on the flamelet equations, which describe turbulent diffusion flames by an ensemble of laminar flame structures 
(Peters, 2000). Under the hypothesis that all chemical species form on a fast-enough time scale, so that their mass fractions and temperature are in quasi-steady state, the temporal derivatives related terms appearing in the full flamelet equations are negligible, so the steady flamelet are described by (Peters, 2000),

$$
-\rho \frac{\chi}{2} \frac{\partial^{2} \phi}{\partial Z^{2}}=\omega
$$

where $\rho$ represents density, $\phi$ is the vector of chemical species mass fractions and temperature, $Z$ is the mixture fraction, and $\omega$ denotes their respective source terms. The scalar dissipation rate of the mixture fraction $\chi$ appearing in Eq. (4) is in turn given by,

$$
\chi=2 D|\nabla Z|^{2}
$$

where $D$ is the mass diffusivity, which is assumed to be equal for all species here. An analytical expression for $\chi$ (Peters, 2000) based on a counter-flow diffusion flame model is used in this work. Notice that the solution of flamelet equations is usually represented using the so-called S-shaped curve, whose upper and lower branches describe the stable burning and non-burning solutions, respectively, and the middle branch the unstable one (Pierce and Moin, 2004). The FPV model utilized here involves an additional flamelet parameter, denoted by $C$, which is a reactive scalar uniquely identifying each single flame state along the $\mathrm{S}$-shaped curve, including the unstable branch. In the present study the reaction progress variable is supposed to be a linear combination of reaction product species, $C=Y_{\mathrm{H}_{2} \mathrm{O}}+Y_{\mathrm{CO}_{2}}+Y_{\mathrm{H}_{2}}+Y_{\mathrm{CO}}$. Similarly, the reaction progress variable source term, denoted by $\overline{\omega_{C}}$, is a linear combination of the chemical source term for the mass fractions of reaction product species, $\overline{\dot{\omega}_{C}}=\dot{\omega}_{\mathrm{H}_{2} \mathrm{O}}+\dot{\omega}_{\mathrm{CO}_{2}}+\dot{\omega}_{\mathrm{H}_{2}}+\dot{\omega}_{\mathrm{CO}}$ (Ihme and Pitsch, 2005). All thermochemical quantities obtained from the solution of the steady flamelet equations are thus parameterized in terms of both the mixture fraction and the progress variable as $\phi=\phi(Z, C)$.

For further evaluation of the filtered quantities, the joint probability density function (PDF) of the two flamelet parameters $\tilde{P}(Z, C)$ is required. The filtered scalar values are then obtained from,

$$
\tilde{\phi}=\int_{C_{\min }}^{C_{\max }} \int_{0}^{1} \phi(Z, C) \tilde{P}(Z, C) d Z d C
$$

And, since $C$ is supposed to be statistically independent from $Z$, the joint PDF is written as,

$$
\tilde{P}(Z, C)=\tilde{P}(Z) \tilde{P}(C),
$$

where the marginal PDF of the mixture fraction is described by a beta-function, which is determined from the mixture fraction mean and variance. For the sake of simplicity, the marginal PDF of $\tilde{C}$ is described by a Dirac delta function (Pierce and Moin, 2004).

Since the range mixture fraction variance $\widetilde{Z^{\prime \prime 2}}$ is related to the $\tilde{Z}$ mean value, a scaled variance of mixture fraction $\tilde{Z}_{\text {eta }}$ defined as,

$$
\tilde{Z}_{e t a}=\frac{\widetilde{Z^{\prime \prime 2}}}{\tilde{Z}(1-\tilde{Z})}
$$

is used as controlling parameter instead, together with $\tilde{Z}$ and $\tilde{C}$.

The FPV model requires the solution of the following transport equations for the filtered mixture fraction $\tilde{Z}$, the SGS mixture fraction variance $\widetilde{Z^{\prime \prime 2}}$ and the filtered progress variable $\tilde{C}$ (Pierce and Moin, 2004),

$$
\begin{aligned}
& \frac{\partial \bar{\rho} \tilde{Z}}{\partial t}+\frac{\partial \bar{\rho} \tilde{u}_{j} \tilde{Z}}{\partial x_{j}}=\frac{\partial}{\partial x_{j}}\left[\bar{\rho}\left(D+D_{t}\right) \frac{\partial \tilde{Z}}{\partial x_{j}}\right], \\
& \frac{\partial \bar{\rho} \widetilde{Z^{\prime \prime 2}}}{\partial t}+\frac{\partial \bar{\rho} \tilde{u}_{j} \widetilde{Z^{\prime \prime 2}}}{\partial x_{j}}=\frac{\partial}{\partial x_{j}}\left[\bar{\rho}\left(D+D_{t}\right) \frac{\partial \widetilde{Z^{\prime \prime 2}}}{\partial x_{j}}\right]+C_{g} \bar{\rho} D_{t}\left(\frac{\partial \tilde{Z}}{\partial x_{j}}\right)^{2}-C_{d} \bar{\rho} D_{t} \frac{\widetilde{Z^{\prime \prime 2}}}{\Delta^{2}}, \\
& \frac{\partial \bar{\rho} \tilde{C}}{\partial t}+\frac{\partial \bar{\rho} \tilde{u}_{j} \tilde{C}}{\partial x_{j}}=\frac{\partial}{\partial x_{j}}\left[\bar{\rho}\left(D+D_{t}\right) \frac{\partial \tilde{C}}{\partial x_{j}}\right]+\overline{\dot{\omega}_{C}},
\end{aligned}
$$


where $D$ is the mass diffusivity, $D_{t}$ is the turbulent diffusivity, $C_{g}$ is a model constant set equal to 2 , and $\Delta$ is the filter width (computed as the cube root of the cell volume). In this work, unity Lewis numbers have been assumed for all species. In addition, $D_{t}$ is modeled according to the relation $S c_{t}=\mu_{t} /\left(\bar{\rho} D_{t}\right)=0.7$, where $S c_{t}$ is the turbulent Schmidt number and $\mu_{t}$ is the turbulent dynamic viscosity. $C_{g}$ in fact is not a free model constant since a theoretical value of 2 is found in the derivation of the exact transport equation (Ihme and Pitsch, 2005), and this value is adopted here. $C_{d}$ is a model constant set equal to 2 , as in (Peters, 2000). Finally, all filtered thermochemical quantities, including $\bar{\rho}$ and $\frac{d}{\dot{\omega}_{C}}$, are retrieved from the steady-state flamelet library parametrized by $\tilde{Z}, \tilde{Z}_{\text {eta }}$, and $\tilde{C}$.

\section{NUMERICAL MODELING}

The numerical modeling approach employed here is described in this section. A particular emphasis is put on the solver and numerical schemes utilized, the spatial and temporal discretization schemes employed, the geometric configuration accounted for, the boundary conditions imposed and the chemical kinetic mechanism used to generate the flamelets.

\subsection{Solver and numerical schemes}

All simulations in this work are carried out with the open source CFD package OpenFOAM, version v2006 (OpenCFD, 2020). In addition, new libraries have been compiled for the flamelet progress variable storage and retrieval algorithms. The employed solver, referred here as 'FPVFoam', is based on the PIMPLE algorithm, a combination of PISO (Pressure implicit with splitting of operator) and SIMPLE (Semi-implicit method for pressure-linked equations) algorithms (OpenCFD, 2020). The workflow associated to this particular solver is shown in Figure 1a. In order to carry out the LES simulations, the transport equations are spatially discretized using a finite volume method (FVM), implicit second-order method Crank-Nicolson with blending factor $\varphi=0.9$ is used for the temporal integration, whereas the convection and Laplacian terms are discretized by second-order accuracy total variation diminishing (TVD) schemes, 'Gauss van Leer' and 'Gauss van Leer corrected', respectively (OpenCFD, 2020). The time step is fixed as $10 \mu$ s obtaining maximum CFL number 0.8 and the steady state is reached at about $300 \mathrm{~ms}$ from the initial state. Averaging of properties starts at $500 \mathrm{~ms}$, corresponding to roughly 5 flow-throughs time based on the fuel jet mean velocity. The averaging process is carried along $1.5 \mathrm{~s}$.

a)

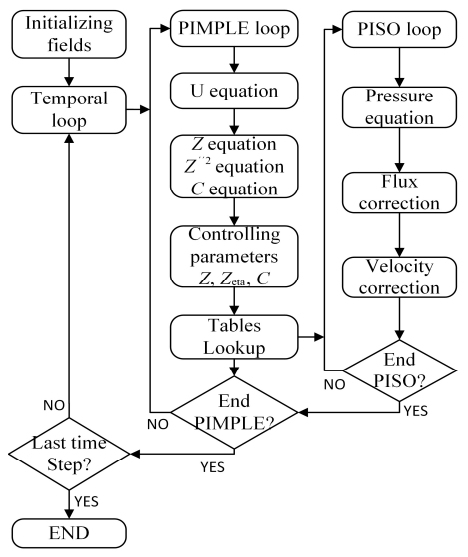

b)

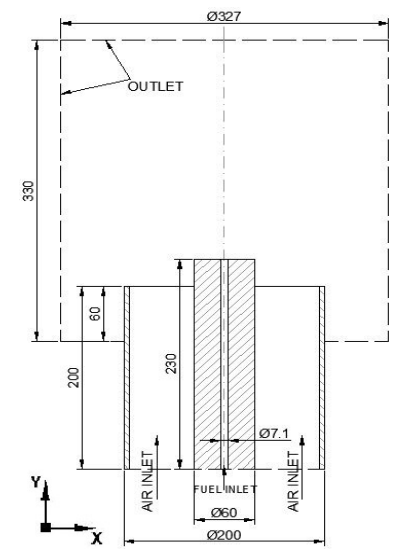

c)

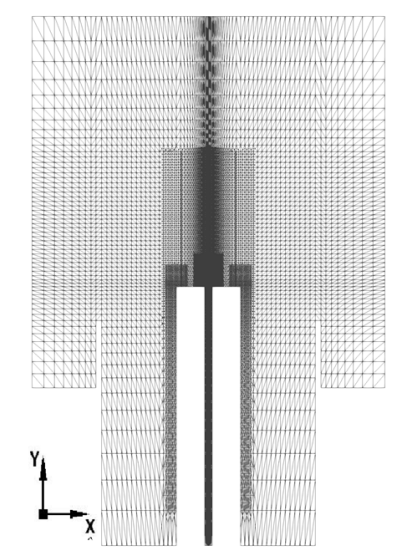

Figure 1. a) FPVFoam solver workflow. b) Geometric configuration, dimensions in mm. c) 3D Computational mesh cross-section.

\subsection{Geometric configuration and mesh generation}

The bluff body geometric configuration and the associated computational domain numerically simulated here have been chosen so as to describe the experimental setup (Caetano and Figueira da Silva, 2015) used as reference in this work. As noticed in Figure 1b, a $200 \mathrm{~mm}$ long annular duct is simulated to describe the air inlet boundary. Similarly, outlets have been placed far enough from the jet discharge in order to avoid their influence on the region of interest, i.e., the bluff body near wake. Other main geometric parameters accounted for such as bluff body diameter $D=60 \mathrm{~mm}$ are also depicted in Figure $1 b$.

The computational domain has been discretized using the OpenFOAM blockMesh utility. An O-grid type mesh has been used to avoid yielding low quality cells near the centerline due to either excessively small cells in the bluff body vicinity or high aspect ratios in the far field. Figure 1c shows the discretized axisymmetric computational domain. The smallest cells have a characteristic size of $120 \mu \mathrm{m}$ at the vicinity of the bluff body lip and wake, whereas the largest cells feature a $5 \mathrm{~mm}$ size at the exit plane farthest radial distance. A y+ value of about 1.5 is ensured at the bluff-body wall, 
whereby the boundary layer of the coflow inlet is expected to be properly described. The computational mesh used here features 4.4 million cells.

\subsection{Boundary and initial conditions}

In accordance with the experimental setup (Caetano and Figueira da Silva, 2015) accounted for here, the fuel jet involved methane $(100 \%)$ at a temperature of $300 \mathrm{~K}$ and a mean flow velocity of $5.3 \mathrm{~m} / \mathrm{s}$ (Reynolds number of about 2428). The coflow stream was characterized in turn by a mean flow velocity of $8 \mathrm{~m} / \mathrm{s}$, a mean turbulent kinetic energy (TKE) of $0.92 \mathrm{~m}^{2} / \mathrm{s}^{2}$, a temperature of $300 \mathrm{~K}$ and a mole fraction composition featuring $79 \% \mathrm{~N}_{2}$ and $21 \% \mathrm{O}_{2}$.

Regarding boundary conditions, velocity is specified at the inlet, the outlet is defined with the standard inletOutlet boundary condition, and for the walls a noSlip condition is set. Concerning pressure, zeroGradient condition is set for the inlets, fixedValue condition of $100 \mathrm{kPa}$ for the outlet, and zeroGradient condition for the walls. The eddy viscosity is set to calculated at both inlet and outlet, and zeroGradient for walls. Filtered mixture fraction has been set to unity and zero at fuel inlet and coflow, respectively. In addition, both mixture fraction subgrid variance and filtered progress variable have been set to zero at both inlets, and zeroGradient condition have been assumed for all walls. In the simulations, a divergence-free synthetic eddy method (DFSEM) (Poletto et al., 2013) has been used at the coflow inlet boundary to reproduce the turbulent inlet coflow measured in the associated experiment.

\subsection{Chemical kinetic mechanism}

The steady flamelet equations are solved using the FlameMaster code (Pitsch, 1998). The gas-phase chemical kinetic mechanism used for this purpose is the GRI-Mech 3.0 (Smith et al., 2007). This mechanism includes 53 chemical species and 325 reactions. This mechanism has been optimized and extensively validated for methane as fuel and it has been employed in the past in the numerical simulation of different turbulent flames (Xie, 2021).

\section{RESULTS AND DISCUSSION}

In this section the main numerical results obtained in this work are presented and discussed. More specifically, a flamelet solution study performed to verify the quality of the generated flamelets in the preprocessing step is firstly described. Next, velocity, turbulent kinetic energy (TKE) fields, as well as the Reynolds stresses are closely analyzed based on experimental data available. The referred results are discussed in both qualitative and quantitative terms.

\subsection{Generation of flamelets}

In Figure 2a, the full set of temperature-related steady flamelet solutions obtained from Eq. (4) is represented by the so-called S-shaped curve, which represents the stoichiometric flame temperature as a function of the stoichiometric scalar dissipation rate. In the referred figure, three branches of solutions, namely (i) top stable burning branch, (ii) middle unstable branch of partially extinguished states and (iii) bottom branch of completely extinguished states, are identified. The turning point between the upper and middle branches occurs at the stoichiometric scalar dissipation rate corresponding to the quenching limit $\chi_{s t, q}$, approximately equal to $30 \mathrm{~s}^{-1}$. A total of 181 flamelet profiles (100 belonging to the fully burning, 80 to the partially extinguished and 1 to the fully extinguished states) have been provided as inputs to the pre-integration codes generating the look-up-tables of the mean thermochemical quantities accounted for.

As highlighted in Figure $2 b$, the generated flamelet profiles consist of a thin diffusive-reaction zone characterized by a high temperature gradient around the stoichiometric mixture fraction, $Z_{s t}=0.055$. As the stretch $\chi_{s t}$ on the flamelets increases, peak temperatures drop because the heat losses to the outer regions of the reaction zone are larger than the amount of heat released. The flamelets finally extinguish when the stretch rate reaches the quenching limit $\chi_{s t, q}$ resulting in an inert mixing of the reactants. The partially extinguished states identified by dashed lines in Figure $2 b$ provide a smooth transition from fully burning to complete extinction conditions. Other flamelet properties have been analyzed, i.e., chemical species $\mathrm{CH}_{4}, \mathrm{H}_{2} \mathrm{O}, \mathrm{CO}_{2}, \mathrm{H}_{2}, \mathrm{CO}, \mathrm{OH}, \mathrm{O}_{2}, \mathrm{H}, \mathrm{O}, \mathrm{C}_{2} \mathrm{H}_{2}, \mathrm{CH}_{2} \mathrm{O}$ and $\mathrm{HO}_{2}$, but the associated results are not included here for the sake of brevity. It is worth noticing as well that, in order to properly describe the flamelets solution curves, a number of points equal to 160 featuring a maximum change in slope ratio and curvature ratio of 0.2 and 0.3 , respectively, has been utilized. In addition, the number of flamelets (181) employed here has been defined in such a way that the difference between 2 consecutive values for each thermochemical quantity analyzed is less than $2 \%$. 
a)

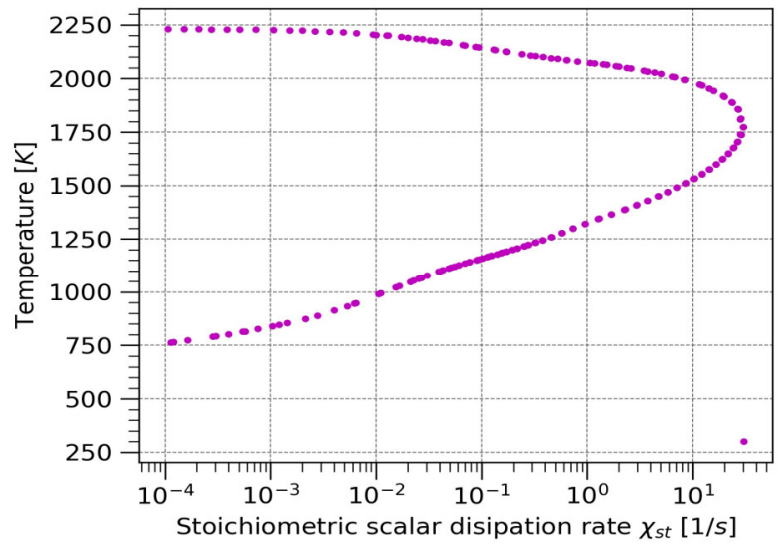

b)

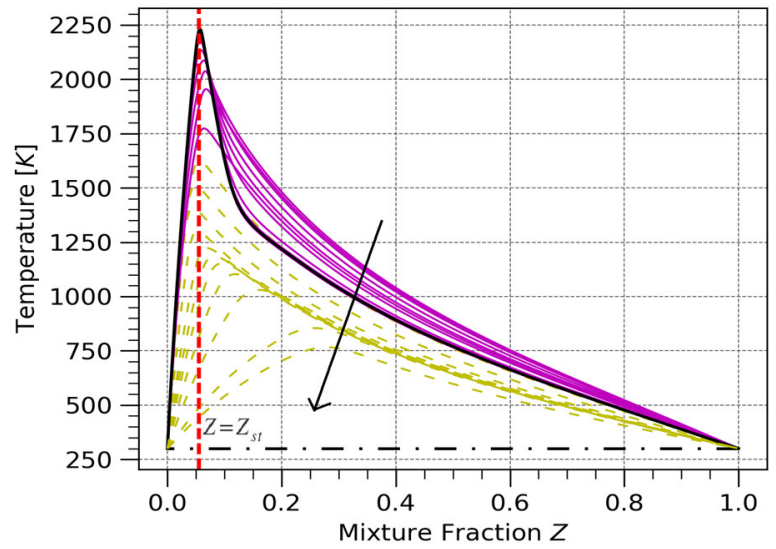

Figure 2. Steady flamelet solutions. Temperature as a function of a) $\chi_{s t}$ at stoichiometric mixture fraction $Z_{s t}=0.055$ and b) mixture fraction (-) fully burning, (- -) partially extinguished and (-.-) fully extinguished states.

\subsection{Mean flow velocity comparison}

Contour plots of computed axial $\left(U_{y}\right)$ and radial $\left(U_{x}\right)$ mean velocities are qualitatively compared to the measured ones in Figure 3. It is noticed from this figure that the computed and measured average flow structure is similar. In particular, the jet penetration depth, the wake spread, and the locations of both the inner vortex close to the fuel jet and the outer one between the inner vortex and the co-flow air are predicted quite well. In quantitative terms in turn, Figure 4 shows that along the centerline $(x / D=0)$ the mean axial velocity behavior is in agreement as well with the experimental data. However, in further downstream near the stagnation point, the central jet velocity does not decrease as fast as in the experiment (Figure 4). The location of the computed stagnation point is thus shifted upstream $(y \approx 80 \mathrm{~mm})$ when compared to the one observed from the experimental data $(y \approx 70 \mathrm{~mm})$ (Figure 4).

In addition, Figure 5 shows a detailed comparison of the mean axial and radial velocity profiles at four different locations corresponding to $y$ equal to $10,30,50$ and $70 \mathrm{~mm}$, where $y$ is the axial distance from the bluff body face. As observed from this last figure, the agreement between predicted results and experimental data is relatively good. At $y=$ $10 \mathrm{~mm}$ for instance, both mean radial and axial velocities agree with the experiments. For all axial locations downstream from $y=10 \mathrm{~mm}$, the location of the minimum mean axial velocity within the recirculation zone $(0.05<|x / D|<0.5)$ also agrees with the experiment. However, the lowest peak of mean axial velocity is significantly over predicted. For instance, at $y=30 \mathrm{~mm}$ the predicted axial velocity is equal to $-0.5 \mathrm{~m} / \mathrm{s}$ but the experimental data indicates $-2.5 \mathrm{~m} / \mathrm{s}$. Also, the maximum and minimum peaks of the mean radial velocity are under predicted but their locations are in general well captured. For the sake of explaining the referred trends, the discussion about the Reynolds stress tensor normal components and the turbulent kinetic energy along the burner center line is developed below.

a)

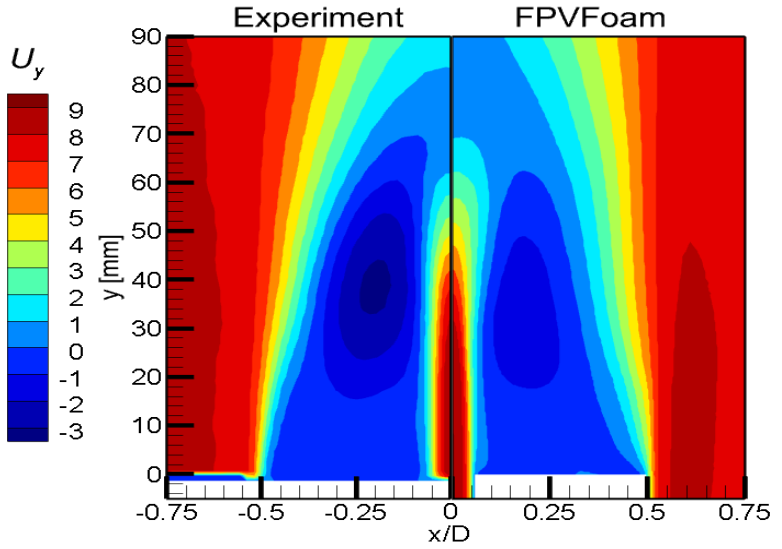

b)

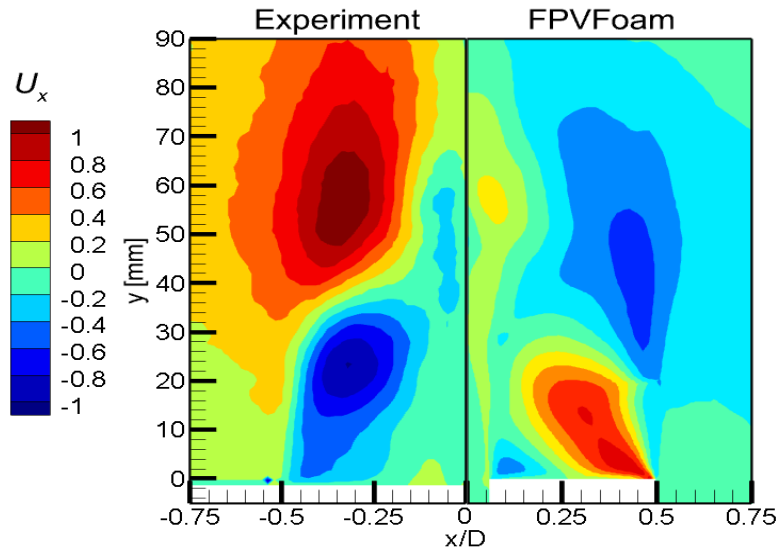

Figure 3. Contour plots of a) axial $\left(U_{y}\right)$ and b) radial $\left(U_{x}\right)$ mean velocities $(\mathrm{m} / \mathrm{s})$. Plots left side: experiment, plots right side: model. 

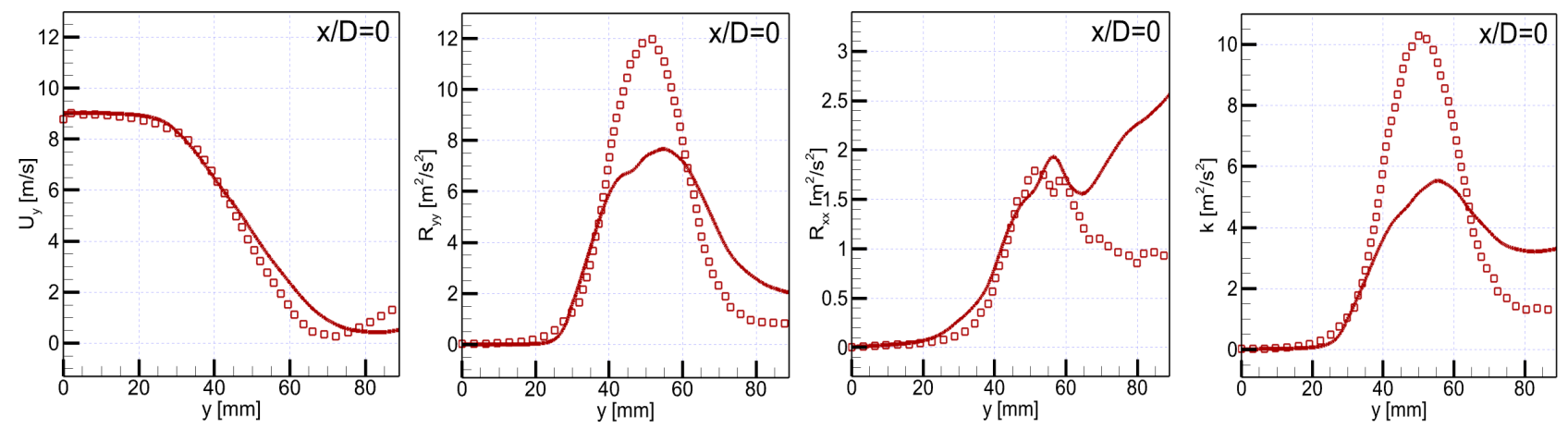

Figure 4. Comparison of computed mean axial $\left(U_{y}\right)$ velocity, Reynolds stress tensor normal components $\left(R_{y y}, R_{x x}\right)$ and turbulent kinetic energy $(k)$ (solid lines) with experimental data ( $\square$ symbols) along burner center line.
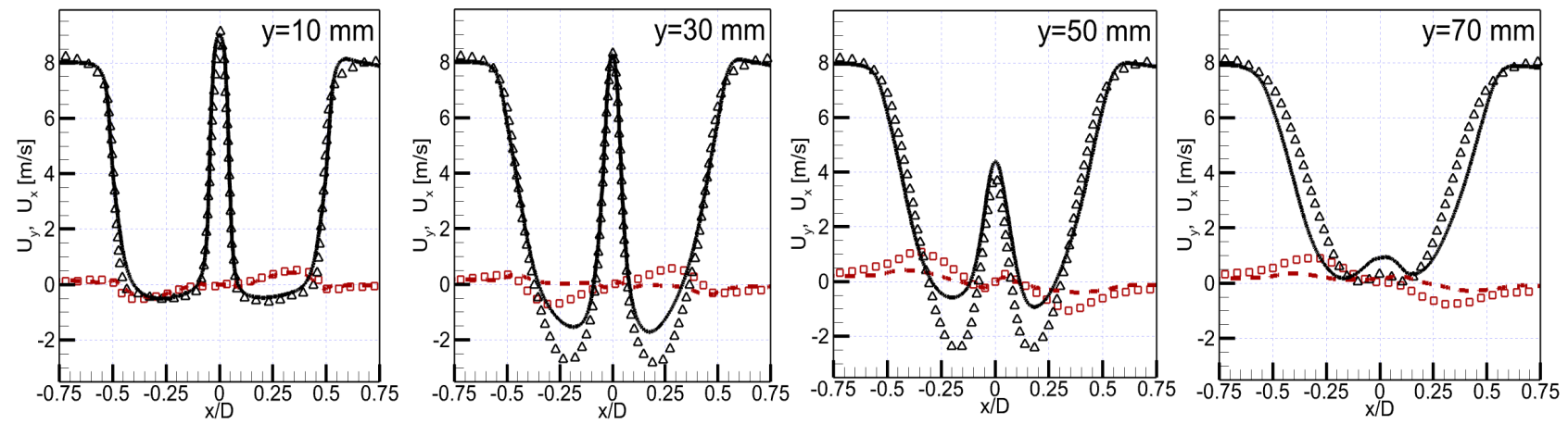

Figure 5. Comparison of computed mean axial $\left(U_{y}\right)$ (solid lines) and radial $\left(U_{x}\right)$ velocities (dashed lines) with experimental results (axial and radial, $\Delta$ and $\square$ symbols, respectively) at different burner axial positions.

\subsection{Reynolds stress tensors and Turbulent kinetic energy}

From Figure 6, which compares in qualitative terms the Reynolds stress tensor components $R_{y y}, R_{x x}$ and $R_{x y}$ and the turbulent kinetic energy $k$, it is observed that the computed flow structure, in terms of $R_{y y}, R_{x y}$ and $k$ is quite similar to the experimental data. Concerning $R_{x x}$ (Figure $6 \mathrm{~b}$ ) until $y=60 \mathrm{~mm}$ a qualitative similar distribution is seen but beyond this point, however, some large discrepancies between numerical and experimental results are noticed. Quantitatively, along the burner centerline, Figure 4 shows that the normal components of the Reynolds stress tensor $\left(R_{x x}, R_{y y}\right)$ and the turbulent kinetic energy $(k)$ present overall similar trends to those characterizing the experimental data. Due to the intense turbulence resulting from the jet breakup within the vortex region, $R_{x x}, R_{y y}$ and $k$ increase up to an axial distance of $y=$ $50 \mathrm{~mm}$, reach a maximum value and then decrease downstream this point. The location of the peak values of $R_{x x}, R_{y y}$ and $k$ at about $y=50 \mathrm{~mm}$ is correctly predicted. The $R_{x x}$ predicted maximum value of $1.8 \mathrm{~m}^{2} / \mathrm{s}^{2}$ is indeed in agreement with the experimental results, but both $R_{y y}$ and $k$ maximum values are under-predicted. From Figure 4 it should be noticed as well that beyond $y=60 \mathrm{~mm}$, predicted $R_{x x}$ tends to increases, whereas in the experimental data this parameter is nearly constant. $R_{y y}$ and $k$ are also over-predicted upstream $y=70 \mathrm{~mm}$ by approximately $1 \mathrm{~m}^{2} / \mathrm{s}^{2}$ and $2 \mathrm{~m}^{2} / \mathrm{s}^{2}$, respectively.

Finally, Figure 7 also highlights the Reynolds stress tensors and the turbulent kinetic energy $k$, but this time at different axial positions. From this figure, it is observed that the $R_{y y}, R_{x x}$ and $k$ profiles at different axial positions agree relatively well with the experimental results, especially for axial locations up to $y=50 \mathrm{~mm}$. The double peaked structure characterizing these turbulent flow properties at each axial position is captured relatively well. Notice that the inner peak corresponds to the inner shear layer, whereas the outer peak corresponds to the outer one. Due to the jet breakup within the inner vortex region, the inner shear layer experiences more production of turbulence and hence greater level of fluctuations than the outer one. Thus, at $y=30 \mathrm{~mm}, R_{y y}$ reaches $8 \mathrm{~m}^{2} / \mathrm{s}^{2}$ in the inner layer whereas a value of $2 \mathrm{~m}^{2} / \mathrm{s}^{2}$ is obtained in the outer one. For all axial positions, the $k$ trends are in agreement with the experiment. Within the recirculation zone, $k$ is over-predicted however at the outer peak and under-predicted at the inner peak. Further downstream $y=70 \mathrm{~mm}, k$ is over-predicted. Regarding the Reynolds stress component $R_{x y}$, Figure $7 \mathrm{~b}$ shows that both the shape and the radial profiles peak values characterizing this tensor component are well captured. The largest values of $R_{x y}$ occur indeed along the boundaries between the fuel jet and the flow inner vortex. 
a)

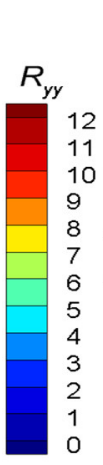

c)
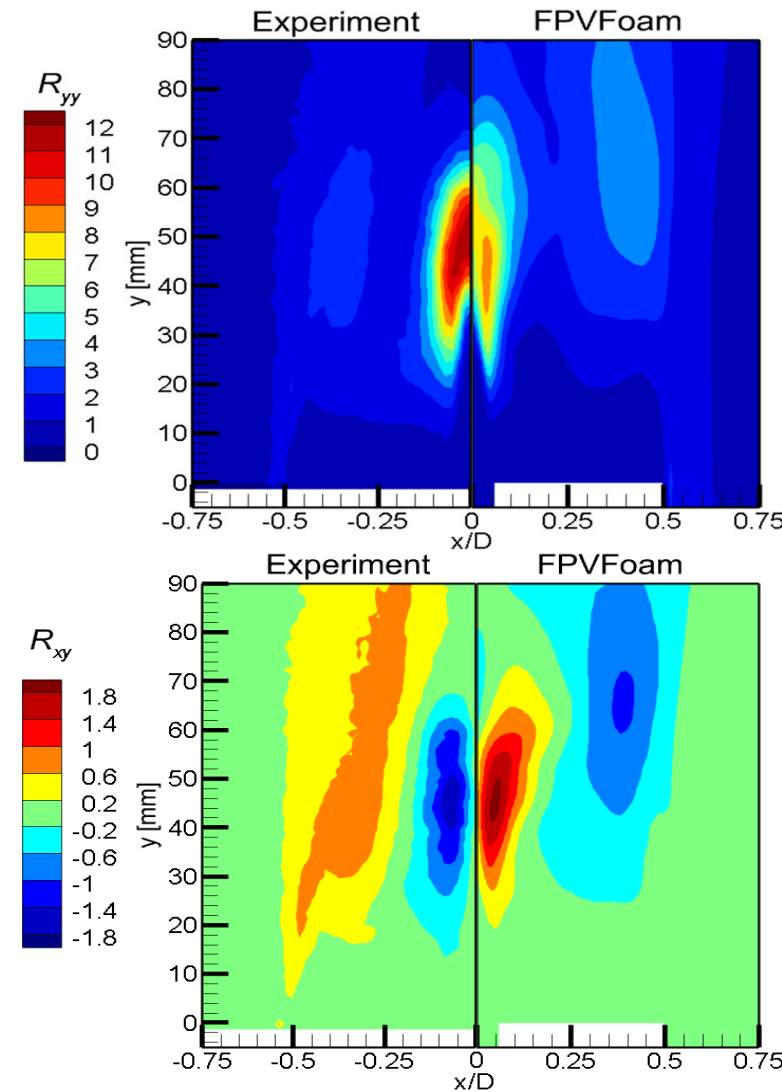

b)

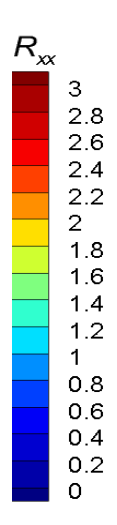

d)
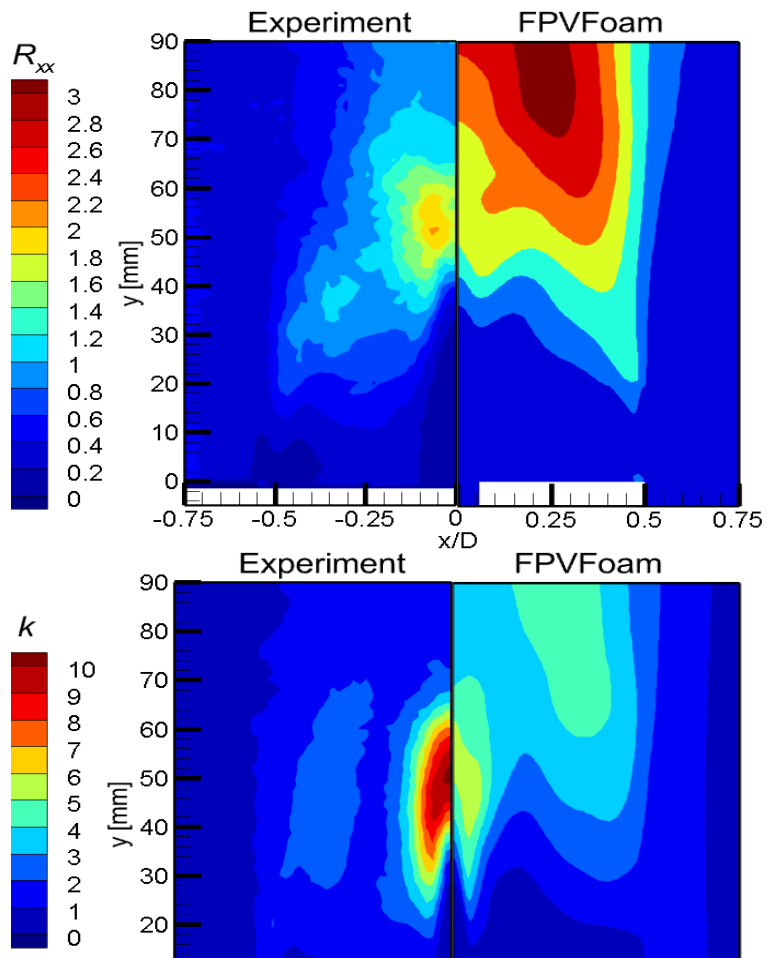

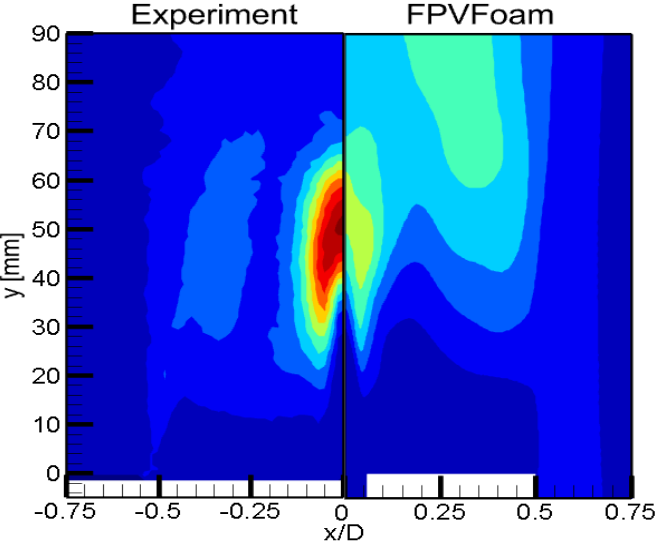

Figure 6. Contour plots of a) $R_{y y}$, b) $R_{x x}$, c) $R_{x y}$ and d) $k\left(\mathrm{~m}^{2} / \mathrm{s}^{2}\right)$. Plots left side: experiment, plots right side: model.

a)

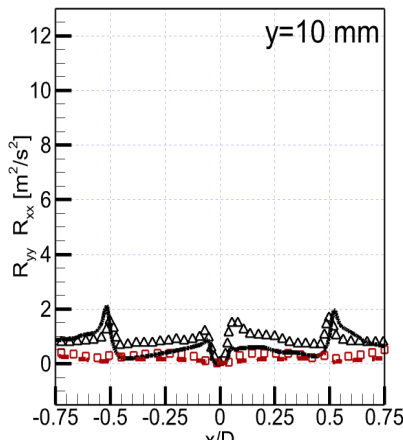

b)

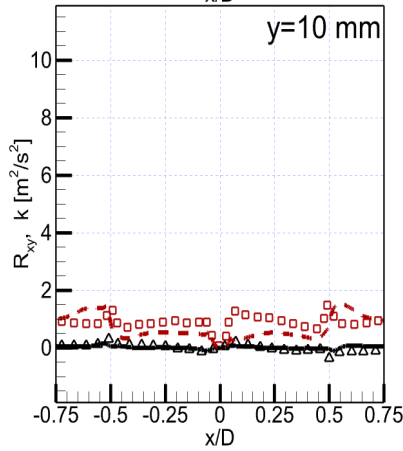

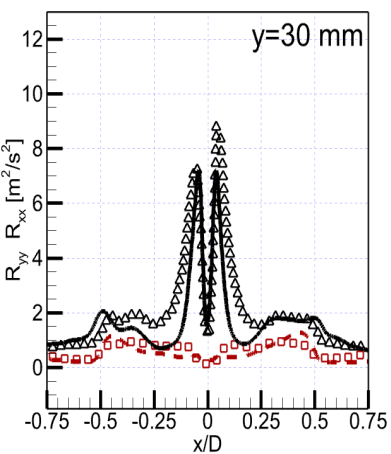

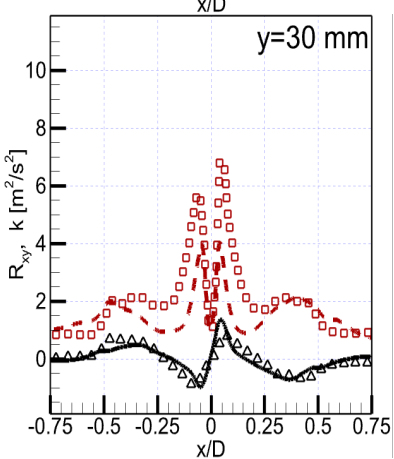

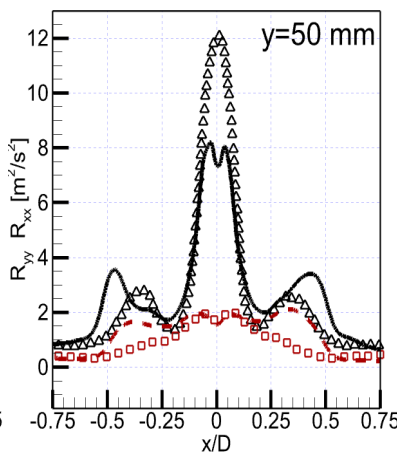

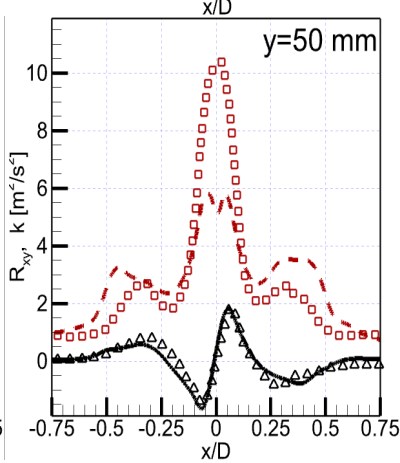

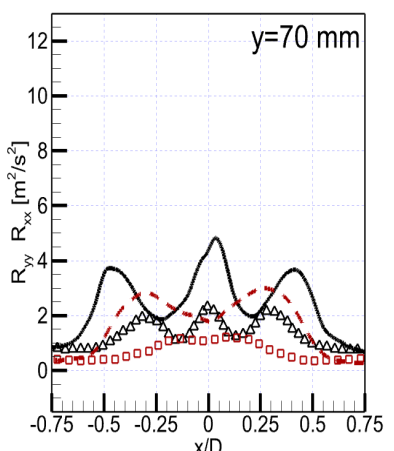

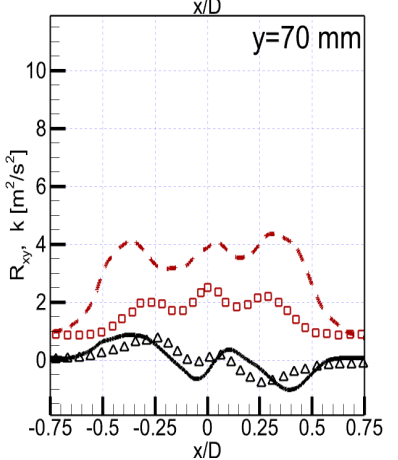

Figure 7. Comparison of computed a) $R_{y y}$ and b) $R_{x y}$ (solid lines) with experiment ( $\Delta$ symbols), and a) $R_{x x}$ and b) $k$ (dashed lines) with experiment ( $\square$ symbols) at different axial positions. 


\subsection{OH distributions and profiles}

Figure 8 shows the instantaneous hydroxyl radical $(\mathrm{OH})$. From this figure it is noticed that there is no a qualitative agreement between the numerical and the experimental results. Indeed, discrepancies between the numerical predictions and the experiments are evident. For instance, in the numerical results, the flame is attached to the burner surface, whereas in the experiment the flame is lifted from the burner and thus a partial premix region is formed upstream the flame edge. In addition, local flame extinctions are not properly predicted as well. In order to deepen the analysis, Figure 9a shows a numerical/experimental comparison of the $\mathrm{OH}$ mean contours overlapped by the corresponding streamlines. Notice that here that $\mathrm{OH}$ fields have been normalized by their maximum values. It is readily observed from this figure that the double vortex structure characterizing the flame is well predicted. The ensemble averaged fields underscore however the significant discrepancies between computed and measured data. For instance, the maximum $\mathrm{OH}$ region is predicted as being close to the burner face, whereas the experiment indicates that this region is located downstream the recirculation zone (Figure 9a). In other words, in the numerical simulations the flame is attached to the burner, which is not the case in the associated experiment. Finally, Figure $9 \mathrm{~b}$ shows radial profiles of normalized $\mathrm{OH}$ at different axial positions. Specifically, at $y=10 \mathrm{~mm}$, compared to the experimental results where values smaller than 0.1 are found, the computed mass fraction of $\mathrm{OH}$ is over-predicted. For all axial positions downstream $y=20 \mathrm{~mm}$, the trends are somewhat similar. However, there are some discrepancies in the $\mathrm{OH}$ maximum values. The $\mathrm{OH}$ maximum peak is over-predicted at $y=30$ $\mathrm{mm}$ and it is under-predicted further downstream, at both $y=50 \mathrm{~mm}$ and $y=70 \mathrm{~mm}$. These discrepancies are related to the fact that the zone of maximum $\mathrm{OH}$ is not downstream of the recirculation zone as in the experimental work used as reference here. Due to the lifted nature of this combustion-related situation, prediction of $\mathrm{OH}$ is particularly challenging. Overall, the OH-related performance of the FPV model employed here highlights the need of choosing a more adequate combustion model for studying this particular burner configuration.

a)

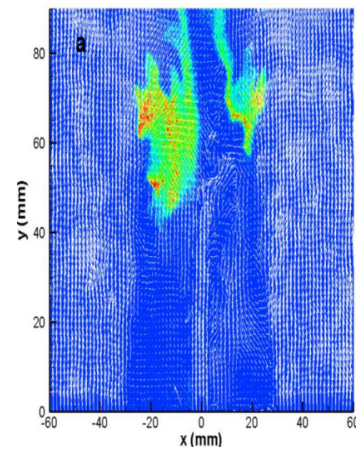

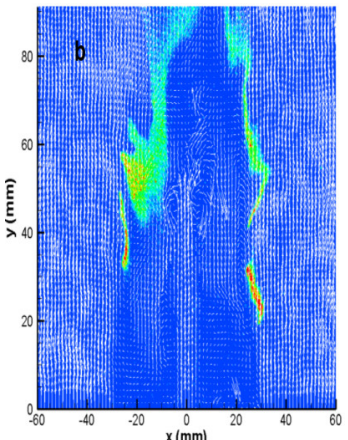

b)

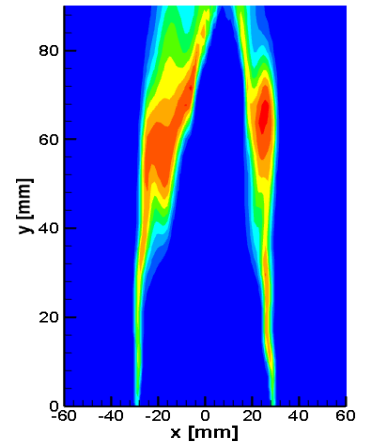

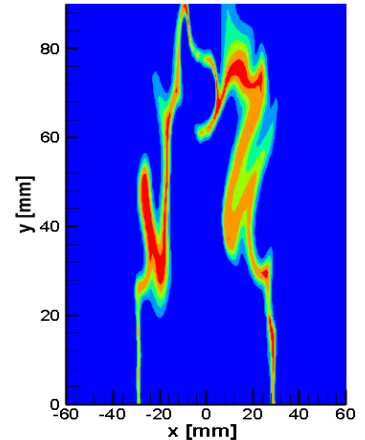

Figure 8. Qualitative comparison of the instantaneous fields of $\mathrm{OH}$ radical. a) Experimental PLIF-OH data, b) computed mass fractions.

a)

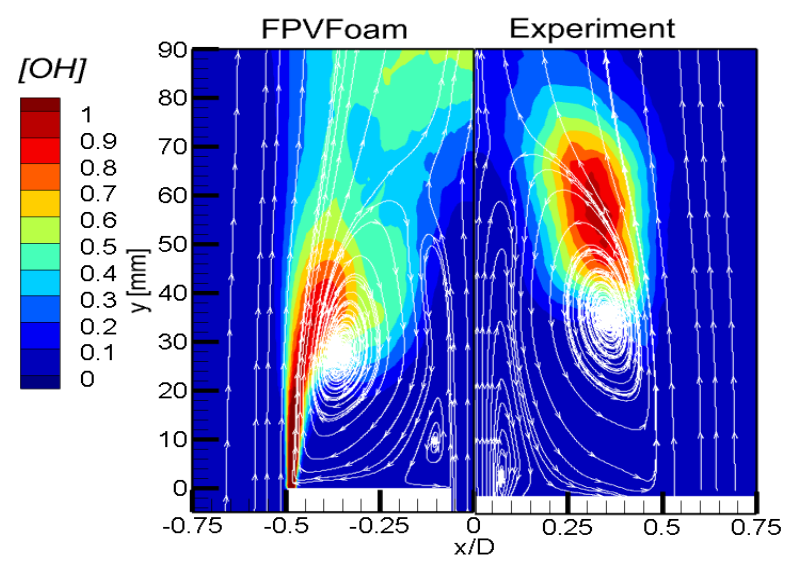

b)

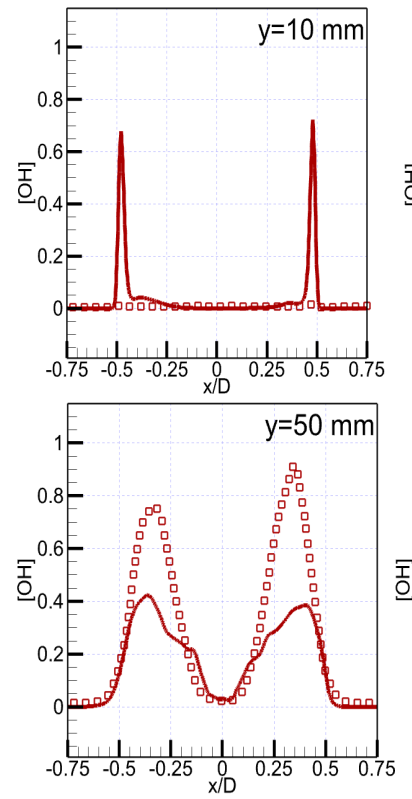

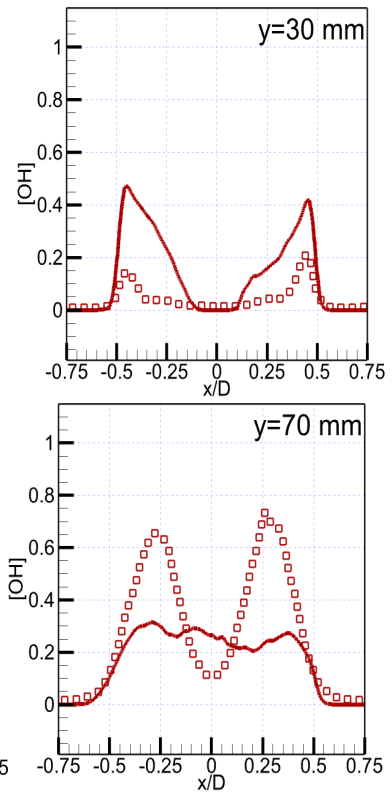

Figure 9. a) Comparison of normalized $\mathrm{OH}$ contours and streamlines. b) Comparison between normalized $\mathrm{OH}$ predicted values (solid lines) and experimental PLIF-OH data ( $\square$ symbols) at different axial positions. 


\section{CONCLUSIONS}

In this work, a flamelet/progress variable (FPV) combustion model was qualitatively and quantitatively assessed using previously measured experimental data characterizing turbulent non-premixed flames stabilized in circular bluff-body burner configurations. GRI-Mech 3.0 chemical kinetic mechanism was used to describe the finite-rate chemistry effects. Flamelets were generated using the FlameMaster code. In order to properly describe the flamelets solution curves, a number of points equal to 160 featuring a maximum change in slope ratio and curvature ratio of 0.2 and 0.3 , respectively, was utilized. In addition, the number of flamelets (181) employed here was defined in such a way that the difference between 2 consecutive values for each thermochemical quantity analyzed is less than $2 \%$. All numerical simulations were carried out in a LES context using OpenFOAM as solver. The numerical results obtained here were compared with the available experimental data using contours and radial profiles at different burner axial positions. Regarding the velocity components, a relatively good agreement between the numerical and experimental results was observed. Both the double vortex structure and the recirculation zone length were well predicted. In addition, Reynolds stresses and turbulent kinetic energy predictions agree with the experiment. Discrepancies downstream the average recirculation zone were observed, however, especially for the Reynolds stress tensor radial component $R_{x x}$. Normalized values of OH radical presented discrepant trends with respect to those associated with the experiment. The obtained results are indeed unable to capture the lifted nature of the measured flame. Intermittently lifted flames present in fact a significant modeling challenge. The final version of the manuscript will further explore the sources of the observed discrepancies. All models developed in this work will be used in a numerical framework currently under development capable of modeling soot formation in combustion systems featuring strong turbulence-chemistry-soot interactions.

\section{ACKNOWLEDGEMENTS}

This work has been supported by CONCYTEC-FONDECYT (Peru), Contract No. 415-2019-2019-FONDECYT, "Identification of soot precursors in turbulent combustion processes through numerical modeling to reduce the impact of soot on both health and environment". During this work Luís Fernando Figueira da Silva was on leave from the Institut Pprime (CNRS - Centre National de la Recherche Scientifique, France). The authors also gratefully acknowledge the support provided by Brazil's Conselho Nacional de Desenvolvimento Cientifico e Tecnologico, CNPq, under the Research Grants No. 304444/2018-9 and 403904/2016-1.

\section{REFERENCES}

Bourdrel, T., Bind, M. A., Béjot, Y., Morel, O. and Argacha, J.F., 2017. “Cardiovascular effects of air pollution”. Archives of Cardiovascular Diseases, Vol. 110, No. 11, pp. 634-42.

Caetano, N.R. and Figueira da Silva, L.F., 2015. "A comparative experimental study of turbulent non premixed flames stabilized by a bluff-body burner". Experimental Thermal and Fluid Science, Vol. 63, pp. 20-33.

Ihme, M. and Pitsch, H., 2005. "LES of a non-premixed flame using an extended flamelet/progress variable model". $43 \mathrm{rd}$ AIAA Aerospace Sciences Meeting and Exhibit.

Lee, C.Y. and Cant, S., 2016. "Assessment of LES subgrid-scale models and investigation of hydrodynamic behaviour for an axisymmetrical bluff body flow”. Flow, Turbulence and Combustion, Vol. 98, pp. 155-176.

Mueller, M.E., Chan, Q.N., Qamar, N.H., Dally, B.B., Pitsch, H., Alwahabi, Z.T. and Nathan, G.J., 2013. "Experimental and computational study of soot evolution in a turbulent nonpremixed bluff body ethylene flame". Combustion and Flame, Vol. 160, pp. 1298-1309.

Nicoud, F. and Ducros, F., 1999. "Subgrid-scale stress modelling based on the square of the velocity gradient tensor". Flow Turbulence and Combustion, Vol. 62, No. 3, pp. 183-200.

OpenCFD, 2020. OpenFOAM: The Open Source CFD Toolbox. OpenCFD Ltd. http://www.openfoam.com.

Peters, N., 2000. Turbulent Combustion. Cambridge University Press, Cambridge.

Pierce, C.D. and Moin, P., 2004. "Progress-variable approach for large-eddy simulation of non-premixed turbulent combustion". Journal of Fluid Mechanics, Vol. 504, pp. 73-97.

Pitsch, H., 1998. FlameMaster, A C++ Computer Program for 0D Combustion and 1D Laminar Flame Calculations. In http://www.itv.rwth-aachen.de/en/downloads/flamemaster/.

Pitsch, H., 2006. "Large-eddy simulation of turbulent combustion”. Annual Review of Fluid Mechanics, Vol. 38, pp. 453482.

Poinsot, T., Veynante, D., 2005. Theoretical and Numerical Combustion. Second Edition: R.T. Edwards Inc.

Poletto, R., Craft, T. and Revell, A., 2013. "A new divergence free synthetic eddy method for the reproduction of inlet 
flow conditions for les". Flow, Turbulence and Combustion, Vol. 91, No. 3, pp. 519-539.

Randers, J., 2012. The Future in Practice: The State of Sustainability Leadership. 2052: A global forecast for the next forty years. pp 1-15.

Rowhani, A., Sun, Z., Medwell, P.R., Nathan, G.J. and Dally, B.B., 2020. "Soot-flowfield interactions in turbulent nonpremixed bluff-body flames of ethylene/nitrogen". Proceedings of the Combustion Institute, pp. 1-8.

Smith, G. P., Golden, D. M., Frenklach, M., Moriarty, N. W., Eiteneer, B., Goldenberg, M., Bowman, C. T., Hanson, R. K., Song, S., Gardiner, W. C., Jr., Lissianski, V. V. and Zhiwei, (2007). In http://www.me.berkeley.edu/gri_mech/.

Valencia, S., Ruiz, S., Manrique, J., Celis, C. and Figueira da Silva, L.F., 2021. "Soot modeling in turbulent diffusion flames: review and prospects". Journal of the Brazilian Society of Mechanical Sciences and Engineering, Vol. 43, No. 219.

Xie, F., Zhou, Y., Song, X., Bai, Y., Wu, R., Yao, M., Yu, G., 2021. "Investigation of OH* chemiluminescence with liftoff characteristic in methane-oxygen inverse diffusion flame”. International Journal of Hydrogen Energy, Vol. 46, No. 2, pp. 1461-1472.

\section{RESPONSIBILITY NOTICE}

The authors are the only responsible for the printed material included in this paper. 